\title{
Physical health assessment and medicines reconciliation on admission to an acute mental health unit: a quality improvement project
}

\author{
Authors: Arani Vivekanantham, ${ }^{A}$ Abdur-Raoof Sheikh, ${ }^{B}$ Hisham Omer, ${ }^{B}$ Patrick Elder ${ }^{B}$ and Samuel Amis ${ }^{B}$
}

\section{Introduction}

Patients with mental health disorders are at high risk of concomitant physical health problems. ${ }^{1}$ Also, medicine reconciliation can help reduce prescribing errors. ${ }^{2}$ The aim of this project was to increase the completion rate of physical health assessments and medicines reconciliations at an acute mental health day hospital in accordance with local/national standards. ${ }^{2,3}$

\section{Methods}

Patient clerking documentation was audited for completion of physical assessments and medicines reconciliation on four occasions: baseline, 2 months after the first intervention, 2 months later (following the trial of an online record-keeping system), and, finally, prior to a third intervention (twice-weekly 'board round' and dedicated weekly 'physical health' clinics) being implemented.

\section{Results}

At baseline ( $n=33), 16(49 \%)$ had a physical examination, 15 (46\%) had an electrocardiogram (ECG), 17 (52\%) had baseline bloods and four (12\%) had a completed medicine reconciliation form. After the second and third intervention ( $n=31)$, these figures increased substantially to $81 \%, 81 \%, 74 \%$ and $74 \%$ respectively.

\section{Conclusions}

Physical health assessments and medicines reconciliation are important components of clerking in psychiatry. The interventions introduced so far have improved the completion rate, thereby improving patient care.

\section{References}

1 Robson D, Gray R. Serious mental illness and physical health problems: A discussion paper. Int ] Nurs Stud 2007:44:457-66.

2 Baxendale L. Procedure for Medicines Reconciliation on Admission to Inpatient Units (V6). Coventry and Warwickshire Partnership NHS Trust, 2016.

Authors: ${ }^{A}$ Arthritis Research UK Centre for Epidemiology, University of Manchester; ${ }^{\mathrm{B}}$ Coventry and Warwickshire Partnership NHS Trust
3 Royal College of Psychiatrists. Occasional Paper OP67. Physical health in mental health. London: RCPsych, 2009. 